J Biomech. 2013 January 18; 46(2): 266-275. doi:10.1016/j.jbiomech.2012.11.032.

\title{
The Syncardia ${ }^{\mathrm{TM}}$ total artificial heart: in vivo, in vitro, and computational modeling studies
}

\author{
Marvin J. Slepian ${ }^{a, b,}{ }^{*}$, Yared Alemu ${ }^{a}$, João Silva Soares ${ }^{a}$, Richard G. Smith ${ }^{c}$, Shmuel \\ Einav $^{b, d}$, and Danny Bluestein ${ }^{a}$ \\ aDepartment of Biomedical Engineering, Stony Brook University, Stony Brook, NY, USA \\ bepartments of Medicine and Biomedical Engineering, Sarver Heart Center, University of \\ Arizona, Tucson, AZ, USA \\ 'SynCardia Systems, Inc., Tucson, AZ, USA \\ ${ }^{\mathrm{d} D e p a r t m e n t}$ of Biomedical Engineering, Tel Aviv University, Tel Aviv, Israel
}

\section{Abstract}

The SynCardia ${ }^{\mathrm{TM}}$ total artificial heart (TAH) is the only FDA-approved TAH in the world. The SynCardia $^{\mathrm{TM}}$ TAH is a pneumatically driven, pulsatile system capable of flows of $>9 \mathrm{~L} / \mathrm{min}$. The TAH is indicated for use as a bridge to transplantation (BTT) in patients at imminent risk of death from non-reversible bi-ventricular failure. In the Pivotal US approval trial the TAH achieved a BTT rate of $>79 \%$. Recently a multi-center, post-market approval study similarly demonstrated a comparable BTT rate. A major milestone was recently achieved for the TAH, with over 1100 TAHs having been implanted to date, with the bulk of implantation occurring at an ever increasing rate in the past few years.

The TAH is most commonly utilized to save the lives of patients dying from end-stage biventricular heart failure associated with ischemic or non-ischemic dilated cardiomyopathy. Beyond progressive chronic heart failure, the TAH has demonstrated great efficacy in supporting patients with acute irreversible heart failure associated with massive acute myocardial infarction. In recent years several diverse clinical scenarios have also proven to be well served by the TAH including severe heart failure associated with advanced congenital heart disease. failed or burnedout transplants, infiltrative and restrictive cardiomyopathies and failed ventricular assist devices.

Looking to the future a major unmet need remains in providing total heart support for children and small adults. As such, the present TAH design must be scaled to fit the smaller patient, while providing equivalent, if not superior flow characteristics, shear profiles and overall device thrombogenicity. To aid in the development of a new "pediatric," TAH an engineering methodology known as "Device Thrombogenicity Emulation (DTE)", that we have recently developed and described, is being employed.

(C) 2013 Published by Elsevier Ltd.

*Correspondence to: Department of Medicine and Biomedical Engineering, University of Arizona, Sarver Heart Center, 1501 North Campbell Avenue, Tucson, AZ 85724, USA. Tel.: +1520626 8543; fax: +1520626 7625. chairman.syns@ gmail.com (M.J. Slepian). Conflict of interest disclosure

Dr Slepian (MJS) and Richard G. Smith are co-founders of SynCardia. There has been no significant financial support for this work that could have influenced its outcome. 
Recently, to further our engineering understanding of the TAH, as steps towards next generation designs we have: (1) assessed of the degree of platelet reactivity induced by the present clinical 70 cc TAH using a closed loop platelet activity state assay, (2) modeled the motion of the TAH pulsatile mobile diaphragm, and (3) performed fluid-structure interactions and assessment of the flow behavior through inflow and outflow regions of the TAH fitted with modern bi-leaflet heart valves. Developing a range of TAH devices will afford biventricular replacement therapy to a wide range of patients, for both short and long-term therapy.

\section{Keywords}

Artificial heart; Mechanical circulatory support; Heart failure; Platelet reactivity; Computational fluid dynamics

\section{Introduction}

Despite dramatic advances that have occurred in the treatment and prevention of all forms of heart disease, congestive heart failure (CHF) as a disease state in particular, continues to be on the rise (Roger et al., 2012). Heart failure, or the progressive decline in cardiac pump function, with an associated systemic compensatory response, mounted as a homeostatic effort to maintain blood pressure and circulation, may occur due to dysfunction of any component of the heart. As such congestive heart failure is commonly regarded as the "final common pathway" of all forms of heart disease. Worldwide there are more than 20 million patients with heart failure, with greater than 5 million patients alone in the United States (Fang et al., 2008). While great strides have been made in the pharmacologic, electrophysiologic and surgical therapy of CHF, many patients unfortunately continue to progress beyond the limits of these therapies and ultimately need a "pump." While the definitive therapy for advanced heart failure is cardiac transplantation, the limited supply of donor hearts has severely limited this therapy (Pierson Iii and Johnson, 2005).

Mechanical circulatory support (MCS), or the use of therapeutic pump systems as augmentation or replacement devices for cardiac pump function, has emerged as an effective means for supporting the failing heart failure patient awaiting transplantation, as well as offering the potential for a long-term therapeutic alternative to transplantation (Kirklin and Naftel, 2008). Over the years MCS systems have continued to evolve and improve. Ventricular assist devices (VADs) capable of augmenting failing ventricular function, configured as either single or dual ventricular (Bi-VAD) systems, have been developed, providing a range of outputs, typically from 2 to $7 \mathrm{~L} / \mathrm{min}$. Over the past twenty years many forms of total artificial heart (TAH) systems have been developed, though the majority of these have never evolved beyond initial human implant studies. By the early 2000s only two TAH systems had progressed to regulatory level trials: the electrohydraulic TAH (AbioCor) and the pneumatic pulsatile TAH (SynCardia). In human trials the AbioCor system was implanted in only 14 patients with limited survival observed, complicated by a high thromboembolic and stroke rate (FDA document: AbioCor Instructions for use-(Fda document 4149b2 01 abiomed instructions for use, 2005)). This device has never been adopted by the clinical community. On the other hand the pulsatile pneumatic TAH has successfully evolved clinically, emerging as an effective system when full bi-ventricular 
hemodynamic restoration is needed (Slepian et al., 2006). The SynCardia ${ }^{\mathrm{TM}}$ total artificial heart (SynCardia Systems, Tucson, AZ) is a pneumatic, pulsatile, complete bi-ventricular replacement system that is surgically implanted orthotopically following cardiac excision, capable of outputs $>9 \mathrm{~L} / \mathrm{min}$ (Copeland et al., 2003). The SynCar-dia ${ }^{\mathrm{TM}}$ total artificial heart (TAH) is the only TAH, despite forty years of research and development by many groups, that has successfully passed the hurdles of rigorous clinical trials, FDA PMA regulatory approval and CMS reimbursement (Premarket approval application (pma) for the syncardia temporary cardiowest total artificial heart (tah-t), 2004; Summary of safety and effectiveness data, 2004; Artificial heart clinical study approvals, 2012).

The SynCardia ${ }^{\mathrm{TM}}$ TAH is indicated for use as a bridge to transplantation for those patients with irreversible bi-ventricular heart failure at imminent risk of death (Syncardia systems inc. Cardiowest total artificial heart (tah) directions for use, 2004). Most recently the TAH also received the FDA designation as a humanitarian use device (HUD), as a step towards clinical use as a long-term implant as an alternative to transplantation, i.e. for "destination." Therapy (FDA grants HUD designation for syncardia's total artificial heart as a destination therapy, 2012). Beside use for either chronic progressive ischemic or non-ischemic cardiomyopathy, new uses for the TAH are emerging. The TAH has proven effective as a means of salvaging patients with protracted, irreversible cardiogenic shock post-myocardial infarction, for heart failure associated with failed Fontan or other repair procedures for congenital heart disease, for failed cardiac transplants, failed VADs and infiltrative cardiomyopathies, to name a few of the expanding scenarios for use.

Along with this expanded profile of use, new needs have emerged which are stimulating the drive for evolution and enhancement of the TAH design. Specifically, a growing unmet need exists for a smaller, i.e. 50 or $30 \mathrm{cc}$, TAH to accommodate individuals, typically women and children, with body surface area $<1.7 \mathrm{~m}^{2}$, i.e. to $<0.9 \mathrm{~m}^{2}$. Further, with the goal of a TAH aimed at long term use, as a transplant alternative, further optimization of thromboresistance and enhanced durability, beyond the present clinical levels is desirous. New manufacturing demands, resulting from the cessation of manufacture of the Medtronic Hall ${ }^{\mathrm{TM}}$ valve, the valve employed in the present clinical TAH, have also created the need for examining and insuring the robust function of the TAH with available and widely utilized, FDA approved bi-leaflet heart valves.

To begin to address these needs, develop next generation TAH devices and enlist support and interest from the Biomedical Engineering community in the artificial heart, in this paper we describe and discuss engineering, clinical and experimental aspects of the TAH.

Specifically, first we describe the design and mechanism of action of the present TAH. We then review the clinical status and performance of the TAH. Finally we review and summarize active onging investigations examining the thrombo-reactivity, flow characteristics and partial modeling of the TAH including: (i) assessment of the degree of platelet reactivity induced by the present clinical $70 \mathrm{cc}$ TAH via a closed loop platelet activity state assay, (ii) modeling of the motion of the pulsatile mobile diaphragm, and (iii) fluid-structure interactions and assessment of the flow behavior through inflow and outflow regions of the TAH fitted with modern bi-leaflet heart valves. 


\section{Construct and mechanism of action of the TAH}

The Syncardia ${ }^{\mathrm{TM}}$ TAH has evolved from over 40 years of work on the development of total artificial hearts. Over the years multiple designs of TAHs have been put forth by varying investigators, an outline of these devices and their use has been described and is beyond the scope of this paper (Copeland et al., 2003). The SynCardia ${ }^{\mathrm{TM}} \mathrm{TAH}$ evolved from early designs of the work of Kolff, Olsen, Jarvik and others. The SynCardia ${ }^{\mathrm{TM}} \mathrm{TAH}$ as a system consists of three component elements: (1) the ventricles-independent right and left blood pumping chambers implanted intra-thoracically, in the space previously occupied by the native diseased ventricles, (2) drivelines- to connect the TAH ventricles to an external pneumatic driver system and (3) the driver-a pneumatic pumping system allowing coordinated but independent pumping of the TAH ventricles with control over various driving parameters (Fig. 1).

The TAH artificial ventricles are constructed as generally spherical blood chambers, allowing inflow and outflow of blood, in a pulsatile fashion, emulating the cardiac cycle. Each chamber is demarcated by an external housing with a semi-rigid basal aspect to which is connected a driveline, and a superior dome-like aspect containing both the inflow and outflow zones in which are mounted mechanical heart valves (Fig. 2). The base assembly is fabricated from IsoPlast ${ }^{\mathrm{TM}}$, engineered thermoplastic polyurethane. The dome-like blood chamber housing is fabricated from segmented polyurethane solution (SPUS) overlaid on a Dacron ${ }^{\mathrm{TM}}$ Mesh. Multiple layers of SPUS are utilized to fabricate the blood chamber, all applied via sequential pouring over a mold. Fitted within the blood chamber, to separate the flow-generating air pulse from the moving blood, are interlaid diaphragms. The diaphragm construct is composed of four independent SPUS diaphragms organized as follows: 1st the air diaphragm, a 2nd diaphragm, a 3rd diaphragm and the 4th blood-contacting diaphragm. Each diaphragm is separated and lubricated by micro-particulate graphite. This multilayer diaphragm construct provides resilience and toughness to the membrane, which will undergo more than $5 \times 10^{7}$ cycles/year of excursion, i.e. inflation/deflation, in a typical patient. Further, this construct provides added safety with regard to potential modes of failure. If a membrane rent or tear were to occur the presence of multiple backup membranes prevents instant blood-air contact, which could be fatal. In over 2000 ventricles implanted to date, i.e. 8000 membranes in use, six small tears have been detected, all occurring beyond 200 days of implant use, all in a single membrane layer, the inner blood diaphragm. For all of these clinical implants to date no multilayer, air-blood contact mediated failure has ever occurred.

During ventricular filling (diastole) and emptying (systole) the diaphragm moves through a complete excursion from the base to the superior aspect of the blood chamber. As such, as a multilayered polymeric construct, with viscoelastic properties, when the ventricle is fully filled the membrane is partially stretched contacting the base of the chamber. As an air pulse is applied, and the ventricle begins to empty, the membrane moves through a central plane, with mild buckling due to the redundancy of the material, akin to flicking or unfurling of a flag or blanket. As ejection motion continues the membrane again is stretched taut and well opposed to the superior aspect of the blood chamber adjacent to the outflow tract. With the future goal of developing smaller as well as very long term use hearts, an attempt at modeling of the motion of the membrane has begun, to better understand its mechanics, 
dynamics sites of wear and fatigue and associated fluid-structure interactions (see Section 4 below).

A range of driver consoles have been developed for the TAH, including both hospital-based drivers as well as, more recently, portable discharge drivers (Fig. 3). The original hospitalbased driver is a large console, mobile only with assistance, known as the circulatory support system (CSS) or "Big Blue" driver. This console consists of two pneumatic drivers -one primary and one backup, air tanks, transport batteries and an alarm and computer monitoring system. Beat rate, $\%$ systole and left and right driving pressure are manually control. In typical use these parameters may be adjusted in the early post-implant period, however, once the patient approaches stability these parameters are rarely reset. Cardiac output, based on volume of airflow out of the drivelines, as well as trend plots of left-sided and right-sided cardiac output are continuously displayed as is drive pressure for each artificial ventricle. Separate ventricular fill volumes are also continuously displayed.

In operation, the primary driver is set to fully eject blood from each artificial ventricle with each beat. For the right ventricle this is achieved by setting the ejection pressure $30 \mathrm{~mm} \mathrm{Hg}$ higher than the pulmonary artery pressure and for the left ventricle by setting the drive pressure $60 \mathrm{~mm} \mathrm{Hg}$ higher than the systemic pressure. The driver, however, is purposely set so as to not allow the artificial ventricles to fully fill. Filling of $50-60 \mathrm{ml}$ per beat, by initial adjustment of the beat rate and \% systole is optimal. This is a key element in the mode of operation of the TAH. The ventricles are purposely under-filled so as to provide a "cushion" of 10-20 $\mathrm{ml}$ to allow for augmentation of venous return and to prevent pulmonary edema from occurring. As a patient exercises, with concomitant increase in venous return, the ventricle has room to accommodate this volume and augment cardiac output to match increasing exercise demand, functioning effectively as a modified Starling mechanism. As presently designed cardiac outputs of greater than $9 \mathrm{~L}$ per minute may be easily achieved. In the future, with the possibility of incorporated sensors and feedback systems, additional mechanisms may be available to modulate and prevent overfilling of the ventricle, as well as for allowing an even greater range of cardiac outputs to be achieved. Presently a new modern, streamlined, digital hospital replacement driver system has recently, come online in Europe for clinical use known as the "Companion 2." This system is much smaller and more mobile than "Big Blue" and is undergoing regulatory review in the United States as well. The Companion 2 will soon replace the "Big Blue" driver worldwide.

The major revolution in driver technology that has completely changed the functional clinical effectiveness of the TAH as a true therapeutic system has been the development of the "Freedom driver." The freedom driver is a small, "lunch pail-sized" portable driver that utilizes a scroll compressor, which may be carried in a backpack or on a shoulder sling (Fig. 4-right). This system has allowed TAH implant patients, once recovered, to leave the hospital and go back to their home environment. This driver has provided complete ambulatory freedom to patients, with the majority resuming active walking, gym activities with some returning to work as well. With the presence of dual battery backup this system allows patients to be unplugged for several hours. A clinical trial examining successful discharge of from the hospital of TAH patients supported by the Freedom driver has recently completed enrollment and is presently being submitted for regulatory approval. Already in 
development is a second generation, robust, lighter weight and quieter "Freedom 2" driver, which is expected to enter clinical use in 2013.

\section{Clinical experience and current status of the TAH}

Clinical experience with the TAH may be best understood if one considers three periods of use: (1) initial early use, (2) the pivotal FDA pre-market approval trial experience and (3) current, post-approval multi-center use.

\subsection{Early TAH use}

The initial clinical use of the TAH use was accomplished at single centers around the world, specifically at the University of Arizona in Tucson, and at the Hopital La Pitie de Salpetriere in Paris. From 1993-2002 62 patients (51 men, 11 women) with irreversible bi-ventricular failure underwent implantation with the TAH at Arizona (Copeland 2004a). Mean LV ejection fraction and CVP pre-implant were $20 \pm 8 \%$ and $20+7 \mathrm{~mm} \mathrm{Hg}$, respectively. The mean time on TAH support was $92 \pm 11$ days (range 1-413 d). Seventy seven percent of patients were successfully bridged to transplantation (BTT), with the TAH. Sixty eight percent of the total group survived to discharge post-transplantation. Twenty three percent of patients died during device support. Multi-organ failure caused $50 \%$ of these deaths. Adverse events included bleeding (20\%), device malfunction (5\%), fit complications (3\%) mediastinal infections (5\%), visceral embolus (1.6\%), and stroke (1.6\%). The linearized stroke rate was 0.068 events per patient-year.

A similar experience was reported by the group from Paris (Leprince 2003). To date the La Pitie-Salpetriere group has the largest experience with the TAH in the world having implanted 208 TAH as of this writing. Between 1986 and 2001, 127 patients (108 males, mean age 38 \pm 13 ) underwent bridge to transplantation with the TAH in Paris. Mean arterial blood pressure and CVP pre-implant were $70 \pm 8 \mathrm{~mm} \mathrm{Hg}$ and CVP $27 \pm 8 \mathrm{~mm} \mathrm{Hg}$ respectively. The duration of support increased progressively in the French experience, averaging 2 months after 1997, with a range from 5 to 271 days. One patient in their early experience was maintained on the TAH for 602 days, due to pre-implantation pre-formed anti-HLA antibodies. Overall $64 \%$ of patients survived to transplantation, with the TAH. Twenty three percent of patients died during device support. Multi-organ failure caused 67\% of these deaths. The clinical thromboembolic event rate they observed was low, with no incidence of CVA and only 2 TIA's. In all they reported on a total experience of 3606 implant-days, with only one instance of mechanical dysfunction. The work of these first adopter centers has been crucial in laying the groundwork for advancement of the TAH as a true effective therapy. The BTT rates achieved by these centers served to demonstrate the capability of full biventricular replacement, achieving results that far surpassed VADs, and to this day remains unsurpassed.

\subsection{Pivotal trial experience}

The most robust multi-center experience published to date with the SynCardia ${ }^{\mathrm{TM}} \mathrm{TAH}$ is the multi-center PMA trial (Copeland et al., 2004b). In this trial the hypothesis tested was that use of the TAH in patients with irreversible bi-ventricular failure would save lives, allowing 
for effective subsequent transplantation. Inclusion criteria for the study were: patients eligible for transplant, NYHA CHF Class IV, BSA range 1.7-2.5 $\mathrm{m}^{2}$, severe hemodynamic insufficiency. From 1993 to 2002 the TAH was implanted in 95 patients (81 protocol, 15 out-of protocol) with irreversible biventricular failure, in imminent danger of death. Major efficacy endpoints included rates of survival to transplantation, overall survival, survival after transplantation and "treatment success," defined as: alive, NYHA Class I or II, not on dialysis or a ventilator and ambulating. A control cohort, of patients, matched with those in the protocol group, without receiving a TAH, was used for contextual comparison.

In this study overall survival to transplantation was achieved in $79 \%$ of patients receiving the TAH versus $46 \%$ of the controls, $p<0.001$. Treatment success was achieved in $69 \%$ of the implant patients versus thirty seven percent of controls, $p=0.002$. The mean time from entry into the study to transplantation or death was 79.1 days for the implant group versus 8.5 days among the controls, $p<0.001$ (Fig. 2). The overall survival rate at one year was $70 \%$ (95\% confidence limit, 63-77\%) in the group receiving an implant as per protocol compared with $31 \%$ in the control group, $p<0.001$ (Fig. 2). Survival at one and five years after heart transplantation was $86 \%$ and $64 \%$, respectively, compared with $69 \%$ and $34 \%$, respectively in the controls. This data compares favorably with the reported overall UNOS survival data of $84.7 \%$ and $69.8 \%$, at one and five years, respectively.

In the multi-center trial significant improvement in secondary endpoints was noted for the TAH group. Patient's hemodynamic status immediately improved following placement of the TAH, with increased systemic pressure, reduced central venous pressure and increased organ perfusion pressure observed. Cardiac index rose from a baseline pre-implant of 1.93.2 L/min $/ \mathrm{m}^{2}$. Renal and hepatic function and the levels of BUN, creatinine, bilirubin and transaminases returned to normal within three weeks of implantation. Electrolyte levels, white count and platelet count also normalized by three to four weeks post-TAH implantation. Quality of life also improved for the TAH group. One week post-implant $75 \%$ of these patients were out of bed. More than $60 \%$ of patients were able to walk more than $100 \mathrm{ft}$. two weeks following implantation.

As with any implantable medical device adverse effects (AE) may be observed. AEs with the TAH were surprisingly reasonable, considering the gravity of the implant. Takeback for bleeding was observed in $28 \%$, with no significant late bleeding observed. Stroke was observed in $4 \%$ of patients, which was lower than that reported for pulsatile VADs. No ascending driveline infection was observed in the pivotal trial.

\subsection{Post PMA approval TAH use}

As a component of the US regulatory approval of the TAH in 2004, the FDA required the subsequent conductance of a post-market approval study. As such over the period from 2007 to 2011100 and 10 patients at 15 medical centers have been enrolled in this study (Slepian et al., 2012). The hypothesis of this trial was that outcomes achieved in the original US Pivotal Trial of the TAH would be "translatable" in the hands of multiple surgeons across varying implanting medical centers in the United States, i.e. that they would achieve similar clinical results. As part of participation in the study a rigorous three-phase training program; including both didactic as well as animal implantation was required. Of the implants 
performed in this study, three centers performed $8-14 \%$ of the implants, eleven centers performing $8 \%$ or less with one center new center, not part of the original pivotal PMA trial group, performing $43 \%$ of the implants. And overall $75.5 \%$ of patients were successfully transplanted compared to $79.9 \%$ of patients in the US Pivotal study ( $p=n s)$. Similarly, the rates of adverse events in the PMSS did not differ from that observed in the Pivotal trial. Thus implantation and use of the TAH in the hands of a broad range of surgeons, many new to this technology, at differing medical centers, appeared readily translatable with equally good outcomes observed.

There has been a noticeable increase in the use of the TAH both, in the US and in Europe over the past two years. At the present time near 100 medical centers are trained or in the process of training to use the TAH. In the first half of 2012, over 50 TAHs have been implanted. This growth is occurring as a result of multiple factors including recognized efficacy of the TAH in cases of severe bi-ventricular failure, superior performance relative to LVADs and Bi-VADs and increasing applicability of the TAH over a wider range of heart failure etiologies, beyond ischemic and non-ischemic cardiomyopathies.

\subsection{Expanding applicability of the $\mathrm{TAH}$}

Within the scope of the present FDA indication for use of the TAH defined pathologies or mechanisms of biventricular dysfunction that are truly well suited for and/or best treated with the TAH have emerged. While traditionally utilized to salvage a patient with progressive decline in bi-ventricular function, in recent years the TAH has been used both in the US and abroad as a very effective acute bailout device for patients with irreversible cardiogenic shock associated with acute myocardial infarction (Slepian and Copeland, 2008). Once stabilized with a rapid deployment MCS bridge to recovery system, e.g. Tandem Heart $^{\mathrm{TM}}$, Impella $^{\mathrm{TM}}$ or ECMO, if ventricular recovery does not occur and the patient is transplant-eligible, the TAH has been employed with good success. The TAH has further been indicated in cases of post-cardiotomy heart failure, with inability to wean patients off cardio-pulmonary bypass.

Aside from use of the TAH in advanced heart failure due to ischemic and non-ischemic cardiomyopathy, the TAH has unique value in several other pathologies and specific situations which are poorly addressed with VADs including: post-transplant heart failure, failing restrictive and infiltrative cardiomyopthies, e.g. cardiac amyloid, failed VAD, and cardiac tumors (see Table 1).

Restrictive and infiltrative cardiomyopathies have emerged as indications in which the TAH may be of great value, as LVAD use in this situation is particularly problematic. Thickened, stiff ventricles present a problem for LVAD inflow cannulae placement and seating. Further, the pathology of these myopathies is associated with reduced ventricular filling. As such VADs are of limited use as the problem is more one of diastolic dysfunction, with reduced filling of the ventricle, as opposed to dilated systolic dysfunction ventricles, better served by VADs. As such ventricular replacement with the TAH in these situations has proven effective and lifesaving. 
Another major emerging indication for the TAH is in the domain of pediatric use. While cardiac surgery has been lifesaving for infants and children born with congential heart defects (CHD), unfortunately for many of these patients that have survived into teenage and young adulthood, failure of these palliative procedures is emerging. As such there is a growing cohort of patients with palliated CHD that are developing end-stage heart failure. The ability to bridge these patients to transplantation with VADs alone is severely limited due to the complex anatomic defects or corrective anastomotic or surgical conduits that have been employed. As such, use of the TAH has recently been demonstrated to be a highly effective means to completely reestablish quality of flow, with full hemodynamic support, reduced morbidity to the patient with successful bridge to transplantation (Morales et al., in press). This growing indication, in particular, is a main driver for developing a next generation smaller TAH.

\section{Ongoing experimental studies-platelet activation state, numerical modeling of the TAH and fluid-structure interaction}

\subsection{TAH platelet activity state studies}

In consideration of advancing the TAH for long-term use, present efforts are underway to more fully understand the platelet activation and thombogenicity of the present design, beyond early work and clinical studies, to examine the possibility of even greater enhancement of its thromboresistance. Whole blood $(120 \mathrm{ml})$ was obtained from healthy adult volunteers $(n=10)$ in accordance with Stony Brook University IRB regulations. The blood was centrifuged at $650 \times g$ for 6 min to separate red blood cells from platelet rich plasma (PRP). Purified platelets in modified Tyrode's buffer were prepared from PRP by gel filtration as previously described (Yin et al., 2004), diluted $\left(2 \times 10^{7} / \mathrm{ml}\right)$ and used for the in vitro flow-loop studies in the TAH described below. Timed samples were analyzed with a modified prothrombinase assay that measures the rate of thrombin generation in direct proportion to shear stress (Jesty and Bluestein, 1999). The resulting platelet activation state (PAS) at various time points and the slope of the fitted line (platelet activation rate or PAR) was quantified for all experiments.

The current FDA approved Syncardia ${ }^{\mathrm{TM}}$ TAH design, constructed with two monoleaflet Medtronic-Hall ${ }^{\mathrm{TM}}$ MHV (25 and $27 \mathrm{~mm}$ ) in each ventricle at inflow and outflow was tested to assess thrombogenicity. Platelet activation was measured using the modified platelet activity state (PAS) assay in a recirculation flow loop consisting of the TAH left ventricleits diaphragm driven by an external reciprocating pump (Harvard Apparatus model 1423) capable of producing quasi-physiological flow curves and regulation of both stroke volume and stroke rate, and a Penrose drain tube connecting the inflow and outflow tracts as previously described (Bluestein et al., 2004) (Fig. 5a), under two representative operating conditions: $95 \%$ ventricular filling at $100 \mathrm{~min}^{-1}$ (cardiac output=6.7 L/min) and at 130 $\min ^{-1}$ (cardiac output $=8.7 \mathrm{~L} / \mathrm{min}$ ). Platelets were sampled every $10 \mathrm{~min}$ for activation measurements as previously described (Yin et al., 2004; Yin et al., 2005).

The platelet activity rate (PAR - the slope of thrombin generation measured over circulation time) for the TAH was found to be $8 \times 10^{-4} \mathrm{~min}^{-1}$ and $17 \times 10^{-3} \mathrm{~min}^{-1}$ when operating at 
heart rates of 100 and 130 beats/min, respectively (Fig. 5b). In comparison to other device components, i.e, the St. Jude Medical bileaflet mechanical heart valve (MHV) alone at $6 \times$ $10^{-4} \mathrm{~min}^{-1}$ (Yin et al., 2004; Yin et al., 2005) this is slightly higher PAR —as expected by the additional components of the TAH. Further, device performance and operating parameters play a significant role in overall thrombogenicity and need to be optimized based on patient-specific cardiac outputs and anticoagulation regimens.

\subsection{TAH computational modeling, fluid-structure interactions (FSI) and computational fluid dynamic (CFD) studies}

As with the empiric studies above efforts are underway to model the TAH using computational methods. With this are efforts to describe the fluid flow through the device using a Lagrangian method which take into account the effect of shear stress accumulation on individual particles, representative of platelets, in the flow field. Fluid structure interaction (FSI) and computational fluid dynamics (CFD) simulations were performed and focus on the left ventricle blood contacting TAH components with the goal of quantifying flow induced stresses that may result in platelet activation through the aortic valve. The TAH simulation model includes the MHV in the aortic position and the left ventricle blood compartment with the deformable diaphragm used to eject the blood through the valve. In the FSI simulations the deformable diaphragm was assumed to be the only moving boundary while the valve was kept in the fully open position, thus representing the systolic ejection phase through the valve.

In the extensive numerical studies described below, an optimization study was performed with the TAH retrofitted with the Medtronic Open Pivot bileaflet mechanical heart valve (MHV) which was chosen as the possible MHV to replace the monoleaflet Medtronic-Hall which is phased out of the market. Of all presently available, FDA approved bi-leaflet valves the Medtronic Open Pivot (formerly ATS) has previously been demonstrated to have more desirable flow characteristics and is the least platelet activating (Dumont et al., 2007; Xenos et al., 2010). Bileaflet MHVs offer better hemodynamic performance but may be more sensitive to their orientation in respect to the flow field. In order to determine the optimal rotational valve orientation which would offer reduced thrombogenic potential for the TAH with this new bileaflet MHV, four TAH geometries with $0^{\circ}, 45^{\circ}, 55^{\circ}$ and $90^{\circ}$ rotational orientations (Fig. 2) were constructed and the resulting platelet stress values were computed and used to compare their thrombogenic performance along multiple flow trajectories.

The ADINA FSI simulation package (ADINA R\&D Inc., MA) was used for the FSI simulations. It utilizes methodology that strongly couples the fluid and solid domains based on large strain and deformation considerations. An arbitrary Lagrangian-Eulerian (ALE) method is used in ADINA to couple the solid and fluid domain deformations. Stress and strain conditions in the solid domain were calculated using the linear dynamics response of a system

$$
M \ddot{U}+C \ddot{U}+K U=R
$$


$M=$ mass, $C=$ damping, $K=$ stiffness matrix, $R=$ external loads, $U=$ displacement, $\ddot{U}=$ velocity and $\ddot{U}=$ acceleration.

Momentum and continuity equations were used to calculate flow and pressure fields in the fluid domain

$$
\begin{gathered}
\nabla V=0 \\
\rho\left(\frac{\partial V}{\partial t}+\left(V-V_{g}\right) \nabla V\right)+\nabla p=\mu \nabla^{2} V
\end{gathered}
$$

$V=$ flow velocity, $\mathrm{v}_{g}=$ local coordinate velocity, $p=$ static pressure, $t=$ time and $\mu=$ viscosity

Blood was modeled as Newtonian fluid with blood properties and an hyperelastic MooneyRivlin rubber material model was used to represent the solid polyolefin rubber of the diaphragm (McMillin, 1987). For the fluid domain, pressure boundary conditions were applied at the membrane-blood interface and downstream of the aortic valve. Non-slip and traction conditions were applied to all solid boundaries, both static and moving. Pressure boundary condition was imposed at the blood non-contacting side of the membrane to deform the solid domain accordingly.

The transient CFD simulations for resolving platelet trajectories and computing the stress loading histories along these trajectories were conducted using FLUENT (ANSYS, Inc. Canons-burg, PA) in the above valve orientations. In these transient CFD simulations the diaphragm motion from the FSI simulations was replaced with imposed boundary conditions representing the diaphragm motion in terms of pressure boundary conditions. The blood was modeled as a two-phase fluid flow with the platelets suspended in plasma assuming laminar flow conditions. Briefly, following mesh independence studies, highly resolved computational grids (approx. 20 million elements) were generated. While some portions of the flow cycle may be characterized as transient turbulent flow, this very highly resolved numerical mesh brings the simulation very close to DNS (direct numerical simulations) which obviates the need to use turbulence models. This simulation approach is further described in detail in our previous publications (Xenos et al., 2010; Alemu et al., 2010).. The two-phase approach computes the coupled interaction of the fluid phase and the particulate, taking into account drag, lift and Basset forces. Spherical $3 \mu \mathrm{m}$ neutrally buoyant particles representing the platelets, were seeded upstream of the valves. Pressure difference was used to drive the two-phase Newtonian fluid through the TAH. Platelet trajectories in the flow field of the TAH and their corresponding stress-loading histories were computed as the combined effect of shear stress and exposure time as previously described (Hellums 1994; Apel et al., 2001; Alemu and Bluestein, 2007) and their stress accumulation distributions computed as an aggregate using the probability density function (PDF) concept described below.

In order to characterize the global thrombogenicity for comparing the various valve orientations, the calculated stress accumulation (SA) information from the staggering number of computed platelet trajectories (up to 30,000 for each case). This information is collapsed into a kernel smoothened probability density function (PDF) of the SA to obtain distribution curves that can be compared side-by-side, coined the "Thrombogenic Footprint" 
(Xenos et al., 2010) specific to each valve orientation. The dominant mode of the thrombogenic footprint identifies the SA range where the majority of the platelets passing through the valves reside in, allowing a side-by-side comparison of different cases. For improved thromboresistance it is desired that the dominant mode of the distribution be shifted to the lower SA range, i.e. a "leftward shift," and as few trajectories as possible residing in the riskier higher SA range (where platelets are likely to activate). PDF thrombogenic footprints of corresponding geometrical changes that improve thrombogenic performance can then be identified.

The PDFs were evaluated by simulations where both single and repeated platelet passages were considered. Single passage calculations-described in detail previously, consider only a single passage during the $300 \mathrm{~ms}$ of the simulated cardiac cycle. Multiple passage platelet stress accumulation calculations were used to obtain values of repeated passages stress accumulation, and are based on the single pass simulations. In these repeated passages simulations a random subset of platelet trajectories were selected from the complete population of the platelet trajectories computed. Other set of random samplings was applied in order to represent consecutive passages through the TAH geometries. This approach represents more realistically repeated passages during consecutive pumping cycles during which the platelets do not necessarily follow the same trajectories, as at each cycle they may randomly follow different trajectories.

In Fig. 7 a freeze frame comparison of the observed and the simulated TAH membrane motion is depicted. As described above, the simulation was performed during the ejection phase when the air compartment is inflated and the blood is ejected through the open aortic valve. Non-uniform membrane motion that resulted in similar mode of buckling is clearly observed in both cases. This membrane deformation dictates the flow conditions through the valves.

Flow accelerates through the valves with a distinctive three jets characterizing blood flow through bileaflet MHV, followed by formation of typical recirculation zones downstream in the wake of the valve. Velocity magnitude pathlines which the platelets follow are presented in Figs. 6 and 8 for the $55^{\circ}$ valve orientation, with peak velocity reaching $1.8 \mathrm{~m} / \mathrm{s}$. These trajectories were used for the stress accumulation (SA) computations and were then used to compute the PDFs, as described above. Stress accumulation was computed for all platelet trajectories extracted from the simulation results (about 30,000 in each case). The multiple passage PDF plot in Fig. 9 shows the comparison of the stress accumulation for flow conditions established by the various valve orientations. The majority of the platelets in the $55^{\circ}$ valve orientation experienced lower maximum stress accumulation values as compared to the other configurations studied.

The SynCardia ${ }^{\mathrm{TM}}$ TAH has emerged as an essential therapeutic device to save lives of patients with irreversible biventricular heart failure. With its expanded clinical use, in the hands of multiple groups around the world, similar successful outcomes like those briefly described herein are being achieved. As this therapy continues to take hold, becoming the standard of care for management of the patient groups outlined above, its use is expected to expand as well, with the TAH becoming a viable means for destination therapy. This is 
particularly the case as a chronic shortage of human hearts available for transplantation exists and will continue into the foreseeable future. With the expected overall growth in the number of patients, achieving the goal of using TAH for long-term therapy mandates optimizing the device both in terms of its performance and thromboresistance. Additionally, unmet needs exist for use of the TAH in smaller patients (BSA $<1.7 \mathrm{~m}^{2}$ ). To meet these needs, state of the art engineering strategies such as the device thrombogencity emulation methodology (DTE) are needed, described herein for improving the performance of TAH by retrofitting it with newer bileaflet MHV and optimizing its thromboresistance. The DTE methodology combines cutting edge numerical simulations of the device and its active components, analysis of entrained blood constituents flowing through the device for quantifying their stress accumulation histories that may lead to blood clot formation, improvement of device design using the DTE methodology in an iterative manner, and proof of the biological consequence of redesigning features through in vitro testing in a hemodynamic shearing device (HSD) and finally platelet activity state (PAS) assessment of the redesigned device prototype

In this study a partial model of the TAH has been developed, including modeling of the motion of the pulsatile diaphragm. In our simulations the pressure applied as a boundary condition for deforming the simulated membrane exceeded that measured in the actual TAH. This boundary condition was artificially increased in order to compensate for the fact that the simulated membrane thickness had to be increased in order to overcome mesh distortion issues. The overall simulation strategy did result in realistic membrane deformation dynamics that correlated well to the recorded video of the TAH membrane dynamics. While the recorded membrane inflation/deflation dynamics indicated several possible buckling modes at various cycles, as the simulations which were conducted for a single cardiac cycle identified only a single buckling mode as expected.

With plans for use of a new valve in the TAH, i.e. an FDA approved bi-leaflet valve, as well as for a reduction in size of the TAH, in this study we specifically examined the issue of the "best orientation," that might be achieved for flow optimization and minimization of therombogenicity. Velocity profiles produced by the four valve orientation simulations show flow dynamics and their corresponding flow stresses that were dictated by the valve's rotational orientation. This resulted in corresponding platelet stress accumulation distribution plots (PDFs) that identified valve orientation offering an optimal thrombogenic performance. Observations from the single pass stress accumulation plots were corroborated with the multipass stress accumulation results, showing distinctive stress accumulation values and their corresponding PDFs for the four TAH valve orientations studied. The PDFs for all cases clearly indicate that the $55^{\circ}$ has the lowest platelet stress accumulation values, i.e., the lowest "thrombogenic footprint" of the various valve orientations studied.

\section{Conclusions}

The SynCardia ${ }^{\mathrm{TM}} \mathrm{TAH}$ is a pneumatically driven pulsatile biventricular replacement device that has demonstrated clear life-saving efficacy in greater than 1000 human implants to date. With increasing adoption of this technology and expanding patient populations that stand to benefit from its use as destination therapy, it may be even further improved through 
thromboresistance optimization. Additionally, engineering demands exists for evolutionary designs that are smaller and utilize new mechanical valves. The DTE methodology is an effective means of rapidly extending and improving the TAH design to create smaller, durable, TAH systems with minimized overall thrombogenicity.

\section{Acknowledgments}

This study was funded by grants from the National Institute of Health: NIBIB Quantum Award Phase I R01 EB008004-01 (DB), and Quantum Award: Implementation Phase II-U01 EB012487-0 (DB).

\section{References}

Alemu Y, Bluestein D. Flow-induced platelet activation and damage accumulation in a mechanical heart valve: numerical studies. Artifical Organs. 2007; 31 (9):677-688.

Alemu Y, Girdhar G, Xenos M, Sheriff J, Jesty J, Einav S, Bluestein D. Design optimization of a mechanical heart valve for reducing valve thrombogenicity-a case study with ats valve. ASAIO Journal. 2010; 56 (5):389-396. [PubMed: 20613492]

Apel J, Paul R, Klaus S, Siess T, Reul H. Assessment of hemolysis related quantities in a microaxial blood pump by computational fluid dynamics. Artifical Organs. 2001; 25 (5):341-347.

Artificial heart clinical study approvals. 2012. 〈http:/www.cms.gov/Medicare/Medicare-GeneralInformation/MedicareApprovedFacilitie/artificialhearts.html $\rangle$

Bluestein D, Yin W, Affeld K, Jesty J. Flow-induced platelet activation in a mechanical heart valve. Journal of Heart Valve Disease. 2004; 13 (3):501-508. [PubMed: 15222299]

Copeland JG, Arabia FA, Tsau PH, Nolan PE, McClellan D, Smith RG, Slepian MJ. Total artificial hearts: bridge to transplantation. Cardiology Clinics. 2003; 21 (1):101-113. [PubMed: 12790049]

Copeland JG, Smith RG, Arabia FA, Nolan PE, McClellan D, Tsau PH, Sethi GK, Bose RK, Banchy ME, Covington DL, Slepian MJ. Total artificial heart bridge to transplantation: a 9-year experience with 62 patients. Journal of Heart and Lung Transplantation. 2004a; 23 (7):823-831. [PubMed: 15261176]

Copeland JG, Smith RG, Arabia FA, Nolan PE, Sethi GK, Tsau PH, McClellan D, Slepian MJ. Cardiac replacement with a total artificial heart as a bridge to transplantation. New England Journal of Medicine. 2004b; 351 (9):859-867. [PubMed: 15329423]

Dumont K, Vierendeels J, Kaminsky R, van Nooten G, Verdonck P, Bluestein D. Comparison of the hemodynamic and thrombogenic performance of two bileaflet mechanical heart valves using a cfd/fsi model. Journal of Biomechanical Engineering. 2007; 129 (4):558-565. [PubMed: 17655477]

Fang J, Mensah GA, Croft JB, Keenan NL. Heart failure-related hospitalization in the US, 1979-2004. Journal of the American College of Cardiology. 2008; 52 (6):428-434. [PubMed: 18672162]

Fda document 4149b2 01 abiomed instructions for use, 2005.

Fda grants hud designation for syncardia's total artificial heart as a destination therapy. 2012. 〈http:// www.dicardiology.com/article/fda-grants-hud-designation-syncardia\%E2\%80\%99s-total-artificialheart-destination-therapy $\rangle$

Hellums JD. 1993 whitaker lecture: biorheology in thrombosis research. Annals of Biomedical Engineering. 1994; 22 (5):445-455. [PubMed: 7825747]

Jesty J, Bluestein D. Acetylated prothrombin as a substrate in the measurement of the procoagulant activity of platelets: elimination of the feedback activation of platelets by thrombin. Analytical Biochemistry. 1999; 272 (1):64-70. [PubMed: 10405294]

Kirklin JK, Naftel DC. Mechanical circulatory support registering a therapy in evolution. Circulation: Heart Failure. 2008; 1 (3):200-205. [PubMed: 19808290]

Leprince P, Bonnet N, Rama A, Léger P, Bors V, Levasseur JP, Szefner J, Vaissier E, Pavie A, Gandjbakhch I. Bridge to transplantation with the Jarvik-7 (CardioWest) total artificial heart: a single-center 15-year experience. Journal of Heart and Lung Transplantation. 2003; 22:12961303. [PubMed: 14672743] 
McMillin CR. Characterization of hexsyn, a polyolefin rubber. Journal of Biomaterials Applications. 1987; 2 (1):3-100. [PubMed: 3504971]

Morales D, Khan M, Gottlieb E, Krishnamurthy R, Dreyer W, Adachi I. Implantation of total artificial heart in congenital heart disease. Seminars in Thoracic and Cardiovascular Surgery. in press.

Pierson RN III, Johnson FL. Evolving role of cardiac transplantation for end-stage congestive heart failure. Transplantation Reviews. 2005; 19 (1):40-46.

Premarket approval application (pma) for the syncardia temporary cardiowest total artificial heart (taht). 2004. 〈http://www.accessdata.fda.gov/cdrh_docs/pdf3/P030011a.pdf〉

Roger VL, Go AS, Lloyd-Jones DM, Benjamin EJ, Berry JD, Borden WB, Bravata DM, Dai S, Ford ES, Fox CS, Fullerton HJ, Gillespie C, Hailpern SM, Heit JA, Howard VJ, Kissela BM, Kittner SJ, Lackland DT, Lichtman JH, Lisabeth LD, Makuc DM, Marcus GM, Marelli A, Matchar DB, Moy CS, Mozaffarian D, Mussolino ME, Nichol G, Paynter NP, Soliman EZ, Sorlie PD, Sotoodehnia N, Turan TN, Virani SS, Wong ND, Woo D, Turner MB. Heart disease and stroke statistics 2012 update: a report from the american heart association. Circulation. 2012; 125 (1):e2-e220. [PubMed: 22179539]

Slepian MJ, Copeland JG. The total artificial heart in refractory cardiogenic shock: saving the patient versus saving the heart. Nature Clinical Practice Cardiovascular Medicine. 2008; 5 (2):64-65.

Slepian, MJ.; Smith, RG.; Copeland, JG. Treatment of advanced heart disease. In: Baughman, KL.; Baumgartner, WA., editors. Fundamental and Clinical Cardiology. Vol. 56. Taylor \& Francis; New York: 2006. p. 473-490.

Slepian MJ, Smith RG, Covington D, Acker M, Arabia FA, Clayson S, Copeland JG, DeValeria PA, Gonzalez-Stawinski GV, Gregoric ID, Long JW, Morris R, Pae WE, Pagani FD, Smedira NG, Smith C, Kasirajan V. Translatable performance with the total artificial heart: the US post-market study. Journal of Heart and Lung Transplantation. 2012; 31 (4):S88-S89.

Summary of safety and effectiveness data. 2004. 〈http://www.accessdata.fda.gov/cdrh_docs/pdf3/ P030011b.pdf $>$

Syncardia systems inc. Cardiowest total artificial heart (tah) directions for use. 2004. (www.fda.gov/ ohrms/dockets/ac/04/briefing/4029b1_final.pdf)

Xenos M, Girdhar G, Alemu Y, Jesty J, Slepian M, Einav S, Bluestein D. Device thrombogenicity emulator (dte) - design optimization methodology for cardiovascular devices: a study in two bileaflet mhv designs. Journal of Biomechanics. 2010

Yin W, Alemu Y, Affeld K, Jesty J, Bluestein D. Flow-induced platelet activation in bileaflet and monoleaflet mechanical heart valves. Annals of Biomedical Engineering. 2004; 32 (8):1058-1066. [PubMed: 15446502]

Yin W, Gallocher S, Pinchuk L, Schoephoerster RT, Jesty J, Bluestein D. Flow induced platelet activation in a St. Jude mhv, a trileaflet polymeric heart valve and a St. Jude tissue valve. Artificial Organs. 2005; 29 (10):826-831. [PubMed: 16185345] 
a

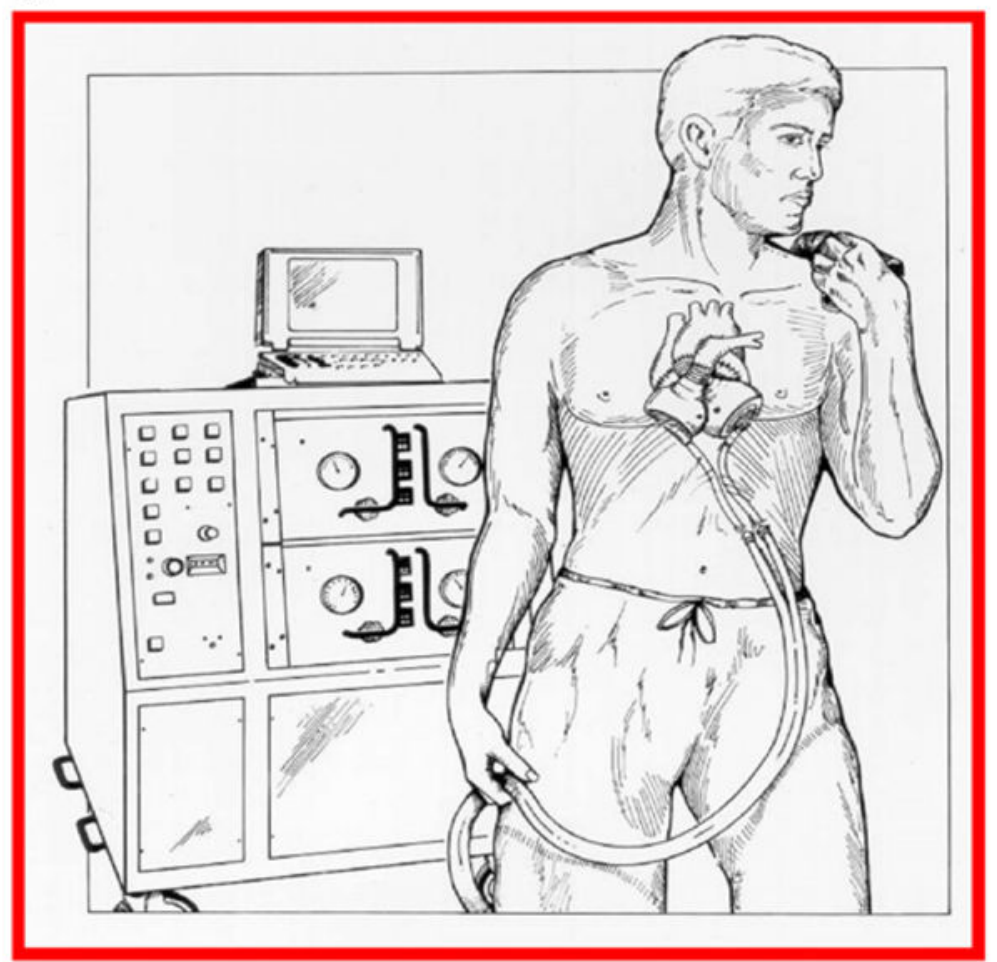

b

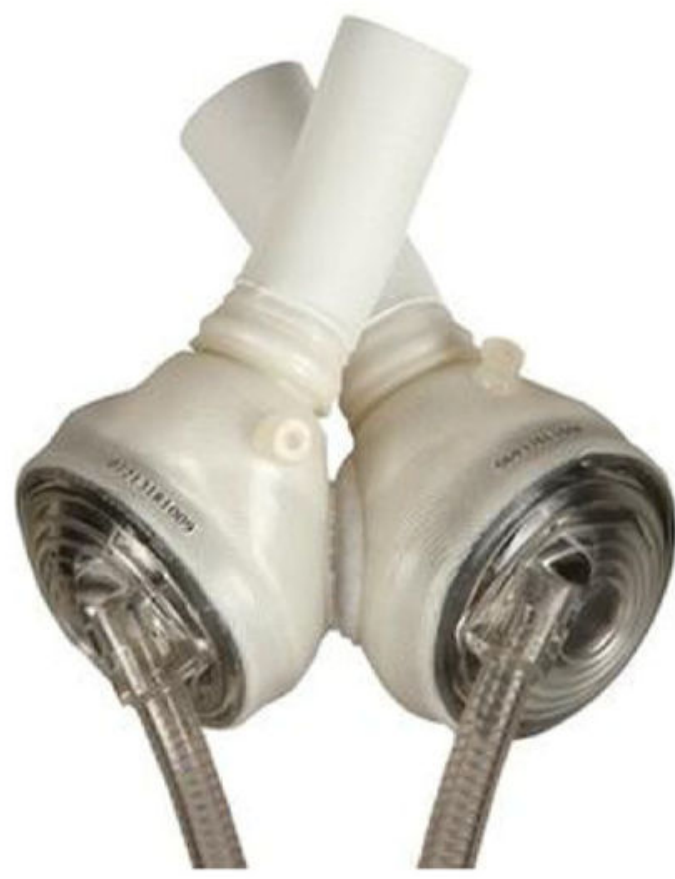

Fig. 1.

Three components of the SynCardia ${ }^{\mathrm{TM}}$ TAH system. (a) Representation of a patient implanted with the TAH. Note right and left ventricles, drivelines and an external driveroriginal "Big Blue" and (b) actual photo of the TAH ventricles with attached outflow grafts and incorporated drivelines. 


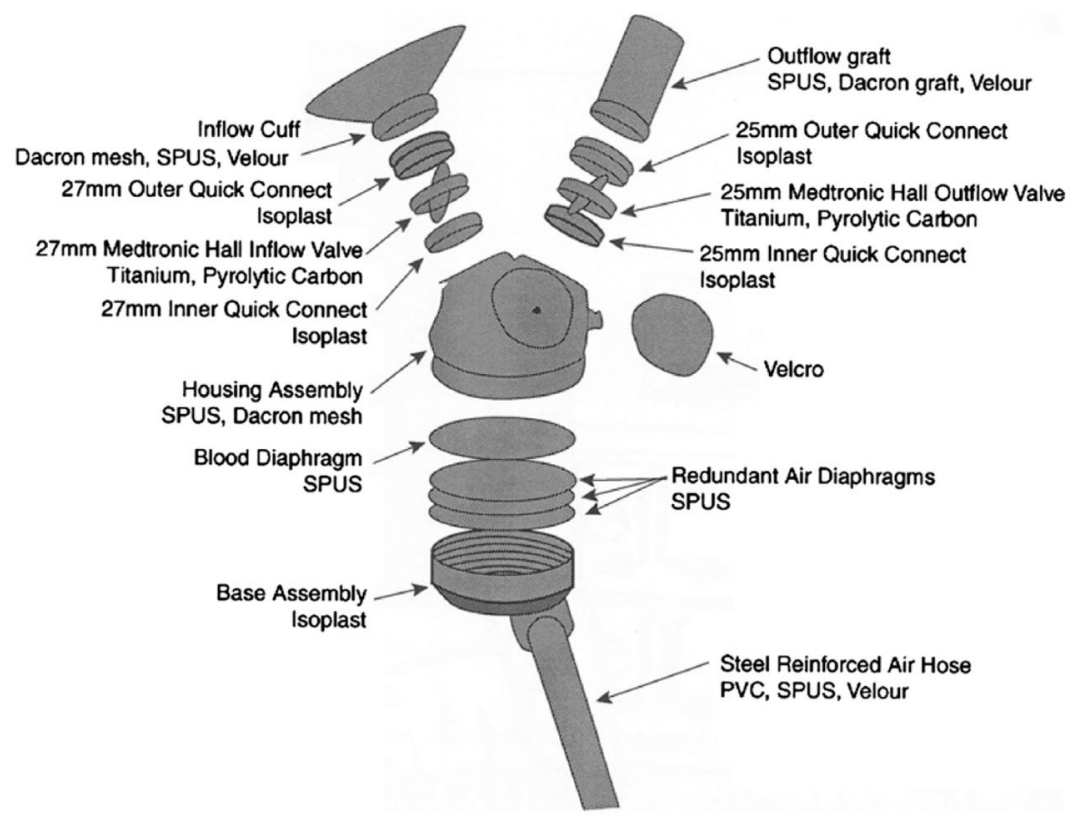

Fig. 2.

Exploded view of SynCardia ${ }^{\mathrm{TM}}$ TAH ventricle. Note housing assembly, diaphragms and base creating separated blood and air chambers. 
a

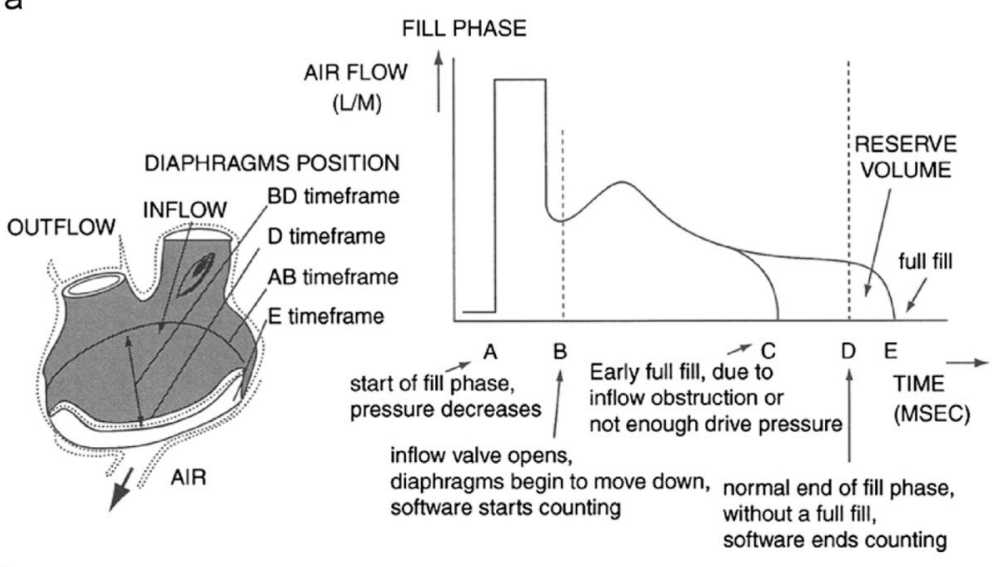

b

EJECT PHASE

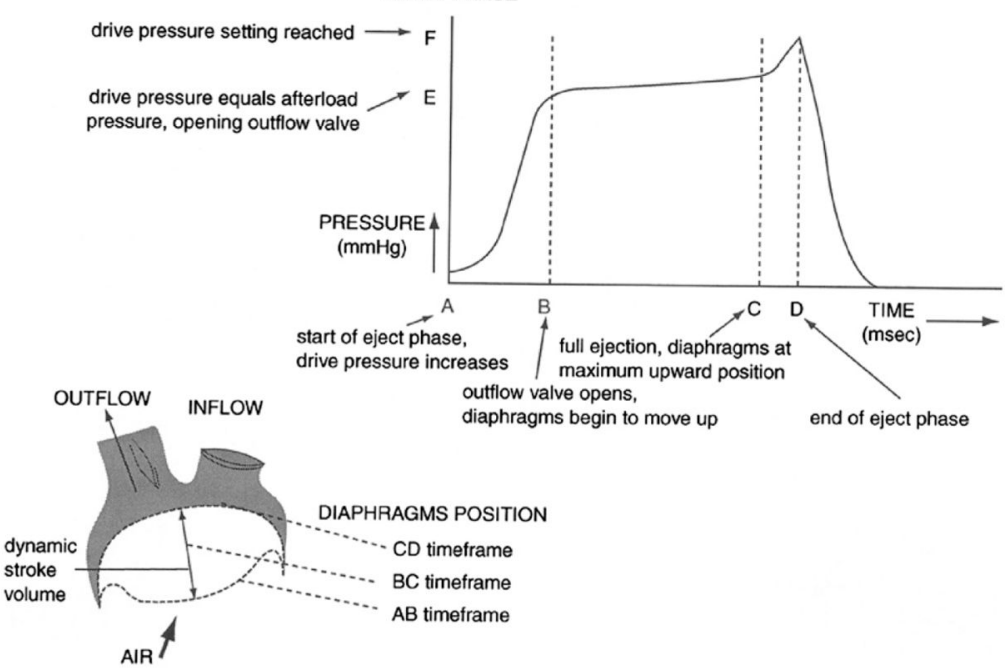

Fig. 3.

(a) Motion of TAH diaphragm assembly and corresponding airflow and pressure waveforms for fill phase and (b) ejection phase. 


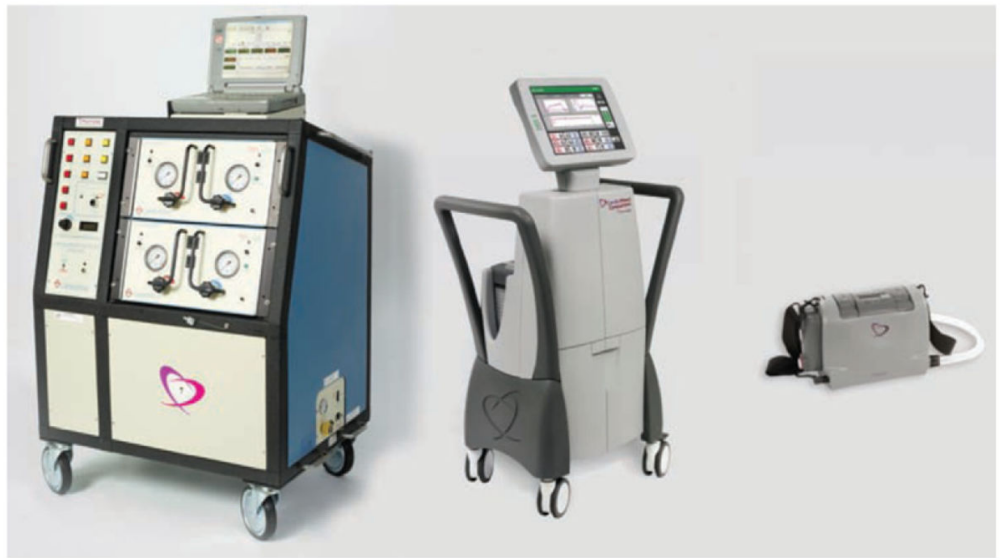

Fig. 4.

Range of pneumatic drivers for the SynCardia ${ }^{\mathrm{TM}} \mathrm{TAH}$. Left—original circulatory support system (CSS) or "Big Blue" driver. Middle—new Companion driver (precursor to Companion 2) and right—Freedom portable "discharge" driver. 
a
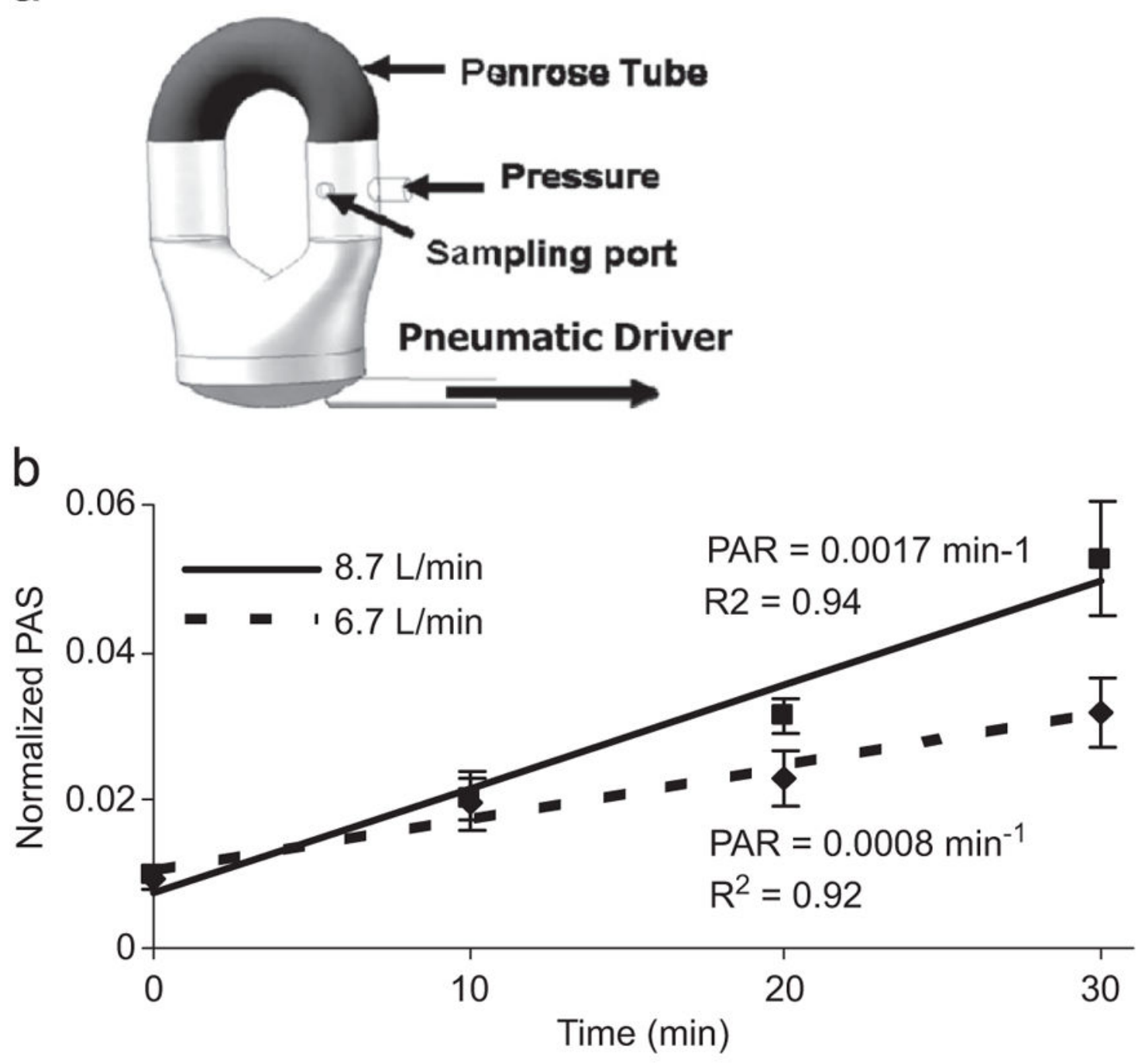

Fig. 5.

(a) SynCardia ${ }^{\mathrm{TM}} \mathrm{TAH}$ left ventricle and a schematic of the flowloop used for the platelet activity measurements and (b) platelet activity rate (PAR) at 6.7 and $8.7 \mathrm{~L} / \mathrm{min}$ cardiac outputs $(p<0.05)$ (bottom). 


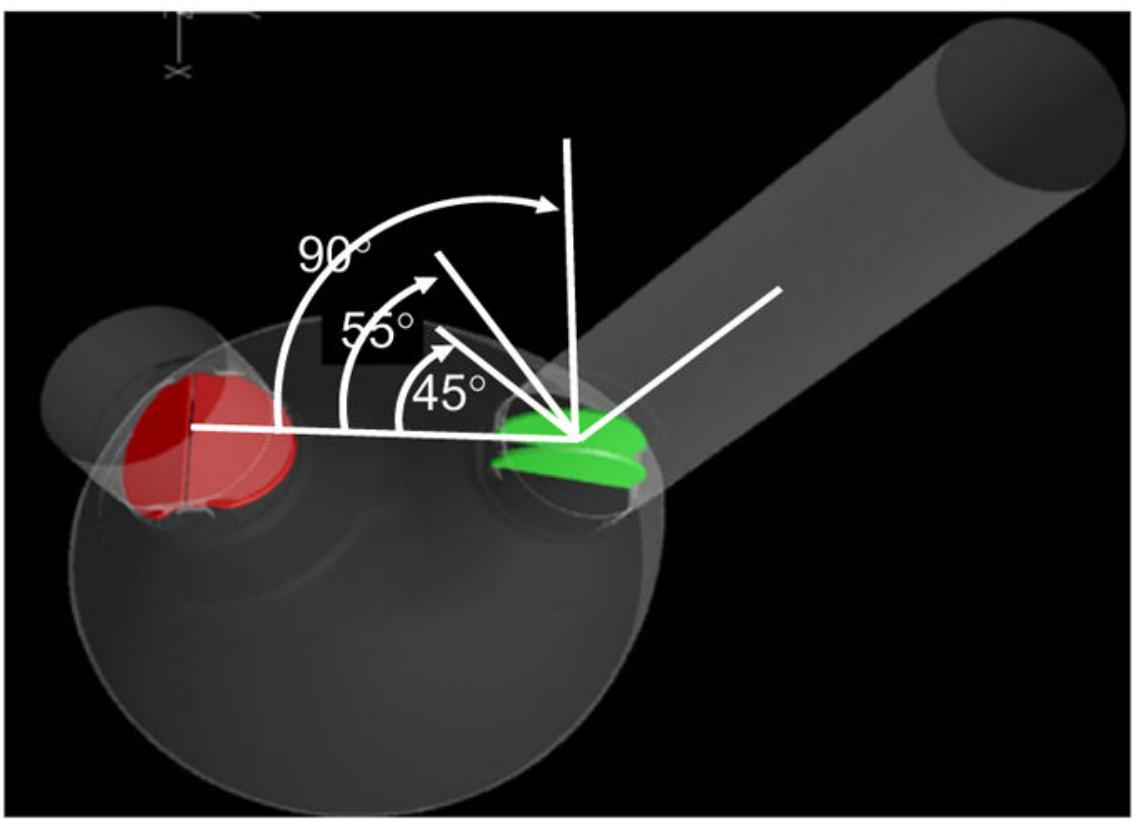

Fig. 6.

SynCardiaTM with open (green) and closed (red) Medtronic Open Pivot bileaflet mechanical heart valves showing the various rotational valve orientations (For interpretation of the references to color in this figure legend, the reader is referred to the web version of this article.) 


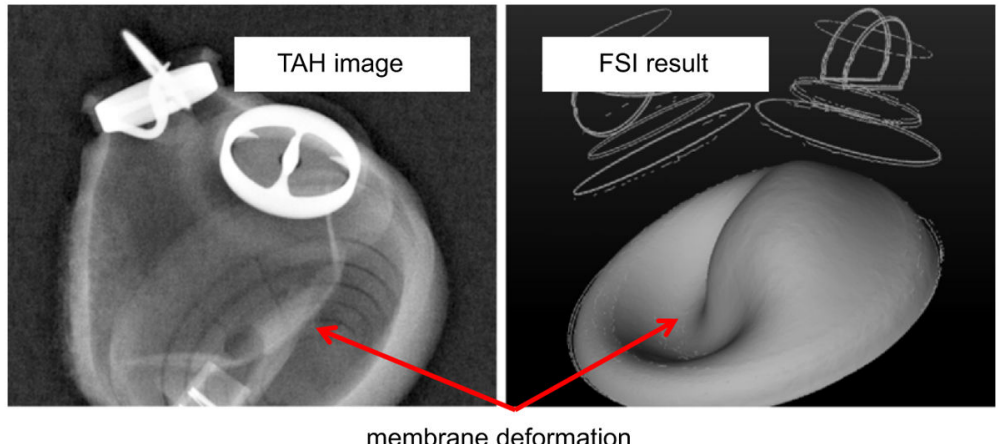

Fig. 7.

Ejection phase of an actual TAH (left) and FSI results (right) showing similar nonuniform deformations of the membrane. 


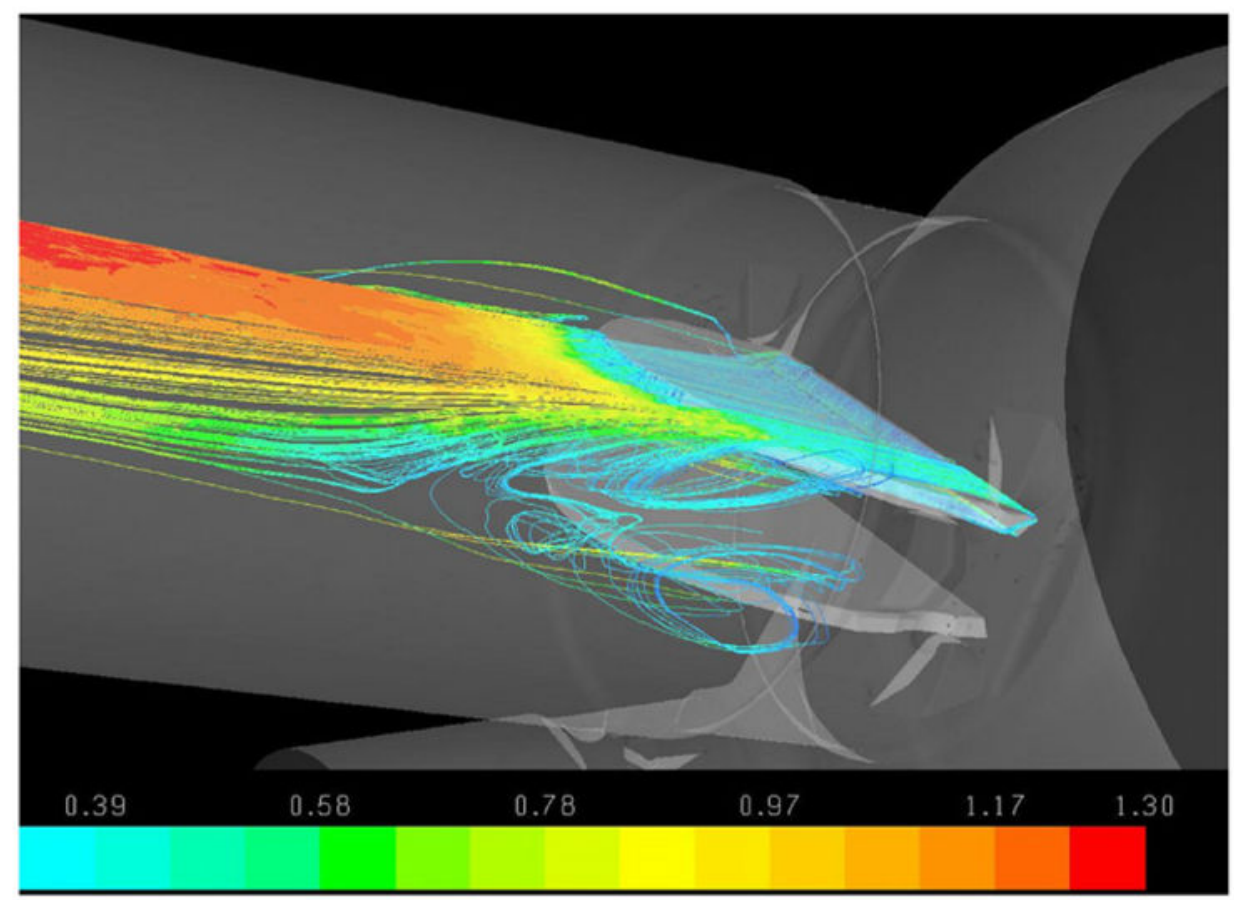

Fig. 8.

Velocity magnitude pathlines—-shown here for the 55O Medtronic Open Pivot MHV orientation. 


\section{Global PDF}

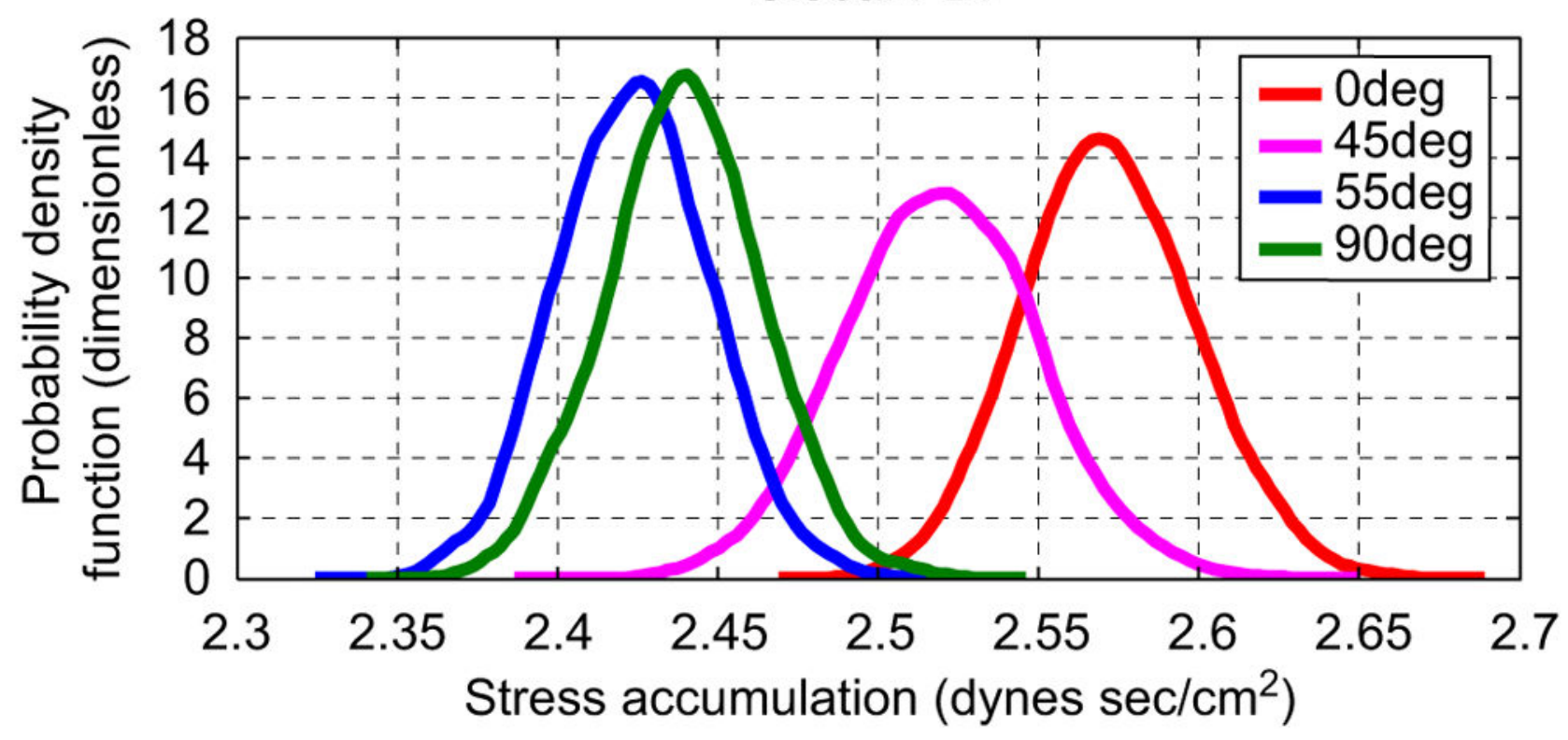

Fig. 9.

Multiple passages PDF ('thrombogenic footprint') for the various valve orientations studied. The "leftward shift" towards lower SA values indicates that the $55^{\circ}$ valve orientation (shown in blue) is the optimal orientation for reduced thrombogenicity (majority of the platelets experience lower flow induced stresses) (For interpretation of the references to color in this figure legend, the reader is referred to the web version of this article.) 


\section{Table 1}

Unique uses for the total artificial heart.

\begin{tabular}{l} 
Severe biventricular failure \\
Massive LV infarct \\
Ventricular thrombus \\
Ventricular septal defects \\
Ventricular rupture \\
Refractory arrhythmias \\
Prosthetic aortic valves \\
Failed cardiac transplant/VAD \\
Adult congenital heart disease—e.g. failed fontan \\
Cardiac malignancies \\
Complex reoperative \\
Infiltrative/restricive cardiomyopathy—e.g. amyloid \\
\hline
\end{tabular}

